\title{
Vulgarisation et diffusion de la médecine pendant la Révolution : l'exemple de la chirurgie*
}

\section{Christelle Rabier}

\section{(2) OpenEdition \\ 1 Journals}

\section{Édition électronique}

URL : https://journals.openedition.org/ahrf/1589

DOI : 10.4000/ahrf.1589

ISSN : 1952-403X

Éditeur :

Armand Colin, Société des études robespierristes

\section{Édition imprimée}

Date de publication : 1 décembre 2004

Pagination : 75-94

ISSN : 0003-4436

\section{Référence électronique}

Christelle Rabier, « Vulgarisation et diffusion de la médecine pendant la Révolution : l'exemple de la chirurgie* ", Annales historiques de la Révolution française [En ligne], 338 | octobre-décembre 2004, mis en ligne le 15 décembre 2007, consulté le 22 avril 2022. URL : http://journals.openedition.org/ahrf/ 1589 ; DOI : https://doi.org/10.4000/ahrf.1589

Ce document a été généré automatiquement le 22 avril 2022.

Tous droits réservés 


\title{
Vulgarisation et diffusion de la médecine pendant la Révolution : l'exemple de la chirurgie*
}

\author{
Christelle Rabier
}

1 Lorsqu'il abandonne la publication de la Gazette de santé à la fin de l'année 1789, le rédacteur justifie sa démarche de la façon suivante: «l'attention publique, qui est entièrement tournée vers les objets du Politique, donne peu d'encouragements pour la continuation d'une feuille entièrement étrangère à ces objets " ${ }^{1}$. Ainsi les événements à l'origine de l'explosion de la presse périodique expliqueraient également, au début de la période révolutionnaire, la disparition d'une des deux feuilles consacrées à la vulgarisation médicale ${ }^{2}$. Le périodique hebdomadaire fondé par Jean-Jacques Gardane, docteur-régent de la Faculté de médecine de Paris, était d'abord «destiné aux gens de campagnes ", avant de s'employer, à partir de 1784, à dénoncer les remèdes des charlatans et à informer ses lecteurs de l'actualité médicale. Après 1789, la Gazette de santé fusionne avec le Journal de Médecine, Chirurgie et Pharmacie, etc. dont la périodicité, mensuelle, satisfait mieux le rédacteur, supposément Philippe Pinel, qui souhaite approfondir les sujets traités. Le projet de diffusion des savoirs médicaux auprès de la notabilité des campagnes, «des curés, des seigneurs, des dames charitables et des fermiers » est abandonné, au profit d'un autre, différent dans sa nature : l'information des « médecins et des chirurgiens de provinces » ${ }^{3}$.

Comme l'a montré Jean-Luc Chappey dans son introduction, la notion de vulgarisation doit faire l'objet, pour l'historien des sciences, d'une attention précise : il ne doit pas seulement étudier les formes et les espaces de la diffusion, mais également comprendre les enjeux de cette "conquête du public ", entreprise qu'on ne peut considérer marginale à la pratique scientifique, où le public serait le destinataire idéal du savoir exposé. De façon à caractériser au mieux les phénomènes de la vulgarisation et de la diffusion médicales, nous nous intéresserons plus particulièrement à l'une des «branches de l'art de guérir », la chirurgie. En effet, à l'époque moderne, la chirurgie est un métier en pleine mutation. Sous la pression de la Couronne, elle évolue de la 
corporation à la faculté, en une discipline qui recouvre des savoirs et des savoir-faire innovants. Ces spécificités permettent d'analyser en finesse les rapports entre les dynamiques sociales et théoriques de la discipline et les enjeux de la vulgarisation.

Réfléchir à la "vulgarisation médicale » à l'époque révolutionnaire implique d'emblée de donner toute sa dimension à "l'art de guérir ». Entre "savoirs et pouvoirs », pour reprendre l'expression heureuse de Jacques Léonard, la chirurgie se caractérise tout autant par l'identité sociale, aux limites floues, de ceux qui la pratiquent, que par ses contenus intellectuels et par ses dimensions économiques. Relevant d'une tradition savante ancienne et d'un métier manuel qui se renouvelle, elle est aussi marchandée auprès des patients. Aussi la vulgarisation à l'oeuvre ne cherche-t-elle pas à toucher uniquement un auditoire, mais aussi une clientèle, si l'on reprend les analyses développées par l'historiographie médicale britannique, héritière des problématiques de J. Habermas sur la sphère bourgeoise et de l'école d'histoire sociale d'E. P. Thompson ${ }^{4}$. Cette remarque liminaire a une conséquence : la conquête du public, à la fois lecteur, spectateur et client - et sa définition même dans la société - concerne à la fois les groupes non-médicaux et la communauté elle-même. Si l'on considère cette acception large, le «public » dépasse les « hommes de lettres » et l'auditoire aristocratique ${ }^{5}$; il concerne également la clientèle qui fait appel aux services des chirurgiens. Quant à la diffusion des savoirs destinée à la communauté des chirurgiens elle-même, qu'on qualifierait aujourd'hui de "formation continue », elle est loin d'être la règle dans la France des Lumières. Jusqu'à la fin du XVIIIe siècle, la réception des chirurgiens s'effectue dans les provinces et ne suppose nullement l'équivalence des formations à l'échelle du royaume. Même après 1749 à Paris et 1756 dans les provinces, dates auxquelles le doctorat devient indispensable à la pratique, les chirurgiens sont loin de former une communauté homogène ${ }^{6}$. Ainsi, au seuil de la Révolution, la question de la construction du destinataire des savoirs de la chirurgie constitue le cœur de la redéfinition disciplinaire qui s'opère au tournant du XIXe siècle, tant en France qu'en Europe.

4 Pour comprendre les enjeux de la diffusion des savoirs chirurgicaux, nous nous fondons sur une production imprimée spécialisée peu nombreuse et sur les paratextes des ouvrages. De plus, les témoignages trouvés dans les archives de l'Académie Royale de Chirurgie, ainsi que dans les inventaires après décès de chirurgiens, permettent d'évaluer le rôle des institutions dans la diffusion des savoirs chirurgicaux.

5 Nous envisagerons dans un premier temps comment la diffusion des savoirs chirurgicaux qui s'accélère à partir du milieu du XVIIIe par l'institution même qui l'a suscitée, l'Académie Royale de Chirurgie. Puis nous analyserons quel fut l'impact de la liberté de la presse sur ce système grippé de publication. Enfin, nous verrons comment, à partir de 1794, soit après la suppression des académies et après la crise qui affecte durement le marché du livre, la publication devient une arme pour redéfinir la communauté chirurgicale sous le patronage de l'État.

I. Entre diffusion et censure, les ambiguïtés de l'Ancien Régime

6 Les premières représentations d'opérations chirurgicales, qu'elles soient picturales ou musicales, disent bien le rôle du chirurgien à la cour, dont l'opération est un spectacle 7 . Si les pratiques spectaculaires existent au début du XVIIIe siècle, tout particulièrement dans les espaces curiaux, tel le Jardin du Roi en 1710 où un public nombreux assiste aux dissections de Dionis, elles ne connaissent plus guère les faveurs du public noble à la fin 
du siècle. L'échec du mesmérisme en est la preuve. Mesmer avait remporté un certain succès dans le monde avant sa condamnation par l'Académie, grâce à des mises en scène autour $\mathrm{du}$ baquet. Le marquis de Puységur, en diffusant sa thérapie somnambulique, les abandonne. En butte aux critiques, il répond par ses Mémoires pour servir à la défense et à l'établissement du magnétisme animal. Dans ce texte qui relate plusieurs thérapies, il en vient à "réserver le spectacle à ceux qui sont disposés à croire ${ }^{8}$. Le magnétisme quitte le débat scientifique public au profit de l'intimité mondaine.

7 Tout au long du siècle, les praticiens médicaux ont utilisé de nombreuses formes de diffusion, proches de la publicité, pour présenter et vendre des médicaments, ou encore des instruments ${ }^{9}$. Pour plusieurs chirurgiens, ce fut d'ailleurs le mode privilégié d'accès à la fortune, voire aux offices curiaux et à la reconnaissance de leurs pairs. Le chirurgien-dentiste François Le Roy de la Faudignière, réputé pour ses collections d'art acquises grâce au « remède ondotalgique » qu'il avait inventé, est devenu chirurgiendentiste du prince palatin des Deux-Ponts ${ }^{10}$. Au cours du siècle, des chirurgiens opèrent des coups de force pour présenter au public des techniques nouvelles. Tel est le cas du Frère Cosme, qui opte en 1748 pour le Journal Historique sur les matières du temps, imprimé à Verdun, lorsqu'il cherche à publier son invention, le lithotome caché, qu'il utilise pour extraire la pierre de la vessie : il a privilégié un périodique très largement diffusé auprès de plus de deux mille souscripteurs ${ }^{11}$. Le chirurgien herniaire Villiers utilise également les ressources de l'imprimé. En 1762, il tente vainement d'échapper au blâme public pour avoir usurpé le titre de correspondant de l'Académie Royale de Chirurgie. En effet, en proposant d'envoyer à l'Académie les affiches qui lui restent, le chirurgien manceau la supplie très humblement :

« de ne pas ordonner que l'extrait de la délibération soit envoyé a l'auteur de la Gazette pour y etre imprimé. je ne vous disimule pas que je suis perdu de cette retractation, tant envers le public qu'en vers MM. nos Medecins de qui je nay nullement la protection ny le suffrage quoyque je naye jamais eus rien de grave a decider avec eux. ${ }^{12}$

8 Les chirurgiens, à titre privé ou collectif, utilisent ici les ressources de l'imprimé, soit pour breveter et vendre une technique ou un instrument, soit au contraire pour contrôler la publication. Bien qu'elle utilise les formes éphémères de l'imprimé, la chirurgie, au milieu du XVIIIe siècle, reste essentiellement véhiculée par les traités et par les périodiques. Les traités recouvrent un spectre large, du manuel édité à plusieurs reprises - tels les Principes de chirurgie de Georges de la Faye - aux ouvrages plus spécialisés, à l'instar des đEuvres de Claude Pouteau. La chirurgie est également présente au sein des publications périodiques généralistes ${ }^{13}$. Leur étude reste à conduire, mais les résultats obtenus pour les périodiques anglo-saxons est probante. Les Philosophical Transactions, comme l'a montré Philip Wilson, constituaient pour les chirurgiens britanniques et continentaux un moyen de faire connaître leurs techniques; les praticiens participaient ainsi activement à l'» entreprise scientifique» de la Royal Society ${ }^{14}$. En Grande-Bretagne encore, alors que la presse spécialisée se développe, il existe jusqu'à la fin du siècle une grande fluidité de circulation entre les périodiques généralistes et médicaux. En 1787, John Hunter, qui anime la Society for the Improvement of Medical and Chirurgical Knowledge, écrit à William Jenner, pour s'excuser du retard pris, car celui-ci lui avait demandé de soumettre une communication sur l'inoculation à la Royal Society, et n'avais pas eu de réponse. "Je m'apprête à la lire ", écrit Hunter, «mais en garderai une copie, car au cas où elle ne 
serait pas publiée dans les Transactions, elle le sera certainement par la Medical Society, qui en fera meilleur usage. ${ }^{15}$

Pour autant, à la veille de la Révolution et jusqu'au début du XIXe siècle, la plupart des chirurgiens français et européens reconnaissent l'importance d'une innovation éditoriale pour la chirurgie : la publication à partir de 1743 des Mémoires de l'Académie Royale de Chirurgie ${ }^{16}$. Marjolin justifie d'ailleurs leur réédition en 1836, par ce qu'ils " pourraient seuls, en quelque sorte, fournir un corps complet de doctrine " ${ }^{17}$. Publié de nouveau à deux reprises au cours des années 1780 par Didot le jeune, ce périodique paraît également sous une forme abrégée et traduit en anglais ${ }^{18}$. Édités par l'Académie Royale de Chirurgie, ils relatent aussi bien l'activité de la société académique que les recherches de ses membres et de ses correspondants. Les Prix, quant à eux, publient de façon périodique les essais qui ont remporté les concours lancés annuellement par la compagnie. En outre, une partie de l'information et des planches du premier volume des Mémoires trouvent une tribune inespérée dans l'Encyclopédie.

En 1753, le Dictionnaire raisonné des sciences et des arts de Diderot et d'Alembert, dans son article "Chirurgie ", affirme avec force l'autorité de la communauté chirurgicale et de l'Académie, dont l'existence est confirmée par lettres patentes en 1748, en proie au milieu des années 1750 à un conflit ouvert avec les docteurs-régents de la Faculté de médecine ${ }^{19}$. Sous la plume d'Antoine Louis, les différentes opérations rendent compte encore davantage du nouveau pouvoir de l'Académie. Au-delà du succès de la nouvelle institution royale et des changements remarquables du statut du chirurgien qui s'ensuivent, il est difficile d'évaluer quel fut l'impact de la large diffusion de l'Encyclopédie sur les savoirs chirurgicaux. On peut même affirmer qu'elle a relativement peu touché les populations chirurgicales. À partir des années 1770 en effet, des dictionnaires de petit format rassemblent les articles de chirurgie publiés dans l'Encyclopédie à destination des praticiens, et ce avant même la parution de l'Encyclopédie méthodique de Panckoucke en $1792{ }^{20}$.

11 Progressivement, le contrôle de l'Académie sur l'édition chirurgicale se fait plus prégnant. Lors de l'affaire de Villiers du Mans, alors que Morand était secrétaire, une relative tolérance existe. «Il est surtout demandé [à Villiers] de ne pas prendre le titre de correspondant de l'Académie dans ses annonces, moyennant quoi il peut faire imprimer ce que bon lui semble ${ }^{21}$. Un tournant se produit entre 1761 et 1766 , avec l'affaire du Journal de Chirurgie. Le 20 juillet 1761, le premier chirurgien du Roi, La Martinière, demande au secrétaire de l'Académie de tout faire pour empêcher l'octroi du privilège à un journal de chirurgie. À ses yeux, « cet ouvrage en embrassant toutes les parties qui sont l'objet des travaux de cette academie, doit necessairement en alterer les sources, et diminuer la correspondance qui fournit matiere à des mémoires " ${ }^{22}$. Le privilège est cependant accordé, comme le fait savoir Morand en séance, lorsque l'Académie refuse l'approbation d'un tel ouvrage. Dès lors, le directeur Pibrac adopte une double stratégie: faire annuler le privilège et éliminer Morand. Devant le chancelier Malesherbes, père du directeur de la Librairie, il avance le même type d'arguments que La Martinière et ajoute que l'Académie, garante du bien public, ne pourra contrôler le contenu du Journal. Il oppose la morale des académiciens et la vénalité des libraires ${ }^{23}$. Quant à Morand, qui semble avoir participé au projet du Journal, il l'accule en exigeant que le secrétaire lui remette l'intégralité des papiers et des mémoires de l'Académie, faisant valoir qu'un secrétaire perpétuel n'était pas inamovible ${ }^{24}$. En 1766, Morand est remplacé par Antoine Louis ; le privilège de librairie 
accordé au Journal de chirurgie est annulé le 28 octobre. Cet épisode de l'histoire de l'Académie est important à plus d'un titre. D'abord, elle consacre le monopole des Mémoires de l'Académie sur la publication périodique française. Par cette jurisprudence ensuite, le gouvernement confie le contrôle de l'édition l'information chirurgicale à l'Académie de Chirurgie et la censure à ses membres. Enfin, elle assigne à l'édition un rôle cardinal dans les pratiques de la chirurgie. De fait, l'Académie devient à partir des années 1760 , le sésame des auteurs à la recherche d'un éditeur parisien. Les archives de l'Académie Royale de Chirurgie conservent certaines demandes d'approbation ${ }^{25}$. Il est vrai que trois postes de censeurs lui reviennent à la Direction de la Librairie, chiffre important lorsqu'on considère que seuls onze savants s'occupent de la médecine, de la chimie et de l'histoire naturelle.

Tous les ouvrages de chirurgie publiés à Paris après 1760 comportent l'approbation du corps savant et le privilège du roi. L'un d'entre eux, Abrégé de l'embryologie sacrée par l'abbé Dinouard, est d'abord publié à l'intention d'un public averti, intéressé par le débat théologique sur le statut de l'embryon. Il prend une nouvelle signification lorsque sa seconde édition, "considérablement augmentée ", et surtout abrégée des commentaires religieux, parait pourvue du «sceau de l'approbation d'un Corps qui peut seul en apprécier le mérite, \& lui procurer la confiance du Public $"{ }^{26}$. Dès lors, l'ouvrage change de nature et devient un traité sur la césarienne, à destination explicite des chirurgiens et des sages-femmes, et non plus des seuls ecclésiastiques et médecins.

Dans la relation qui lie l'auteur et l'éditeur, l'Académie Royale de Chirurgie en vient à jouer un rôle crucial, celui de « procurer la confiance du Public » et de sanctionner ainsi la publication de tout ouvrage de chirurgie. Le public invoqué représente pour Dinouard la clientèle potentielle de l'ouvrage ; il est donc tribunal dont il faut gagner la confiance. La faveur du public est particulièrement nécessaire aux entreprises périodiques, qui soulignent fréquemment son importance ${ }^{27}$. Pourtant, lorsque Pibrac dénonce les libraires et leurs associés qui «ne faisoient cela que pour gagner de l'argent", il oppose le marché et "l'honneur" qui régit l'Académie. Obéissant aux règles morales de la cour, la société savante est garante du bien public. Cessant de juger en dernière instance de la qualité d'un ouvrage, le public devient la dupe, victime des " charlatans ", c'est-à-dire de ceux qui ne reconnaissent pas l'autorité de l'Académie Royale de Chirurgie.

14 Ce pouvoir n'empêche pas la naissance, à partir de 1770, d'une contestation de plus en plus nourrie. En effet, l'impression des Mémoires de l'Académie Royale de Chirurgie cesse après 1774 ; les mémoires qui arrivent nombreux à l'Académie et qui y sont discutés, sont oubliés dans les archives, au grand désespoir des praticiens en quête de reconnaissance. Les traités qui ne trouvent pas grâce aux yeux des académiciens doivent paraître sous le manteau. Louis Antoine Valentin fait paraître son ouvrage « à Amsterdam » supposément ${ }^{28}$. Enseignant au Collège Royal de Chirurgie et membre de l'Académie, il y présente ses travaux sur les plaies de la poitrine avec épanchement, les amputations et le bec-de-lièvre. Il y conteste violemment le pouvoir du secrétaire perpétuel de l'Académie, Antoine Louis, dont « l'autorité et la routine » l'indispose et y redéfinit d'une nouvelle façon les rapports entre l'Académie et le public, toujours mineur. En particulier, il conteste le système de valeurs qui régule les échanges de la chirurgie.

«Je n'ignore pas l'utilité de ces échanges, qui n'en imposent à aucun des parties contractantes, \& dont le public seul est la dupe; mais j’ai déjà observé, en 
commençant, que si un commerce, ou plutôt une charlatanerie de ce genre, étoit excusable dans la simple littérature, dans les sciences d'agrément, elle devenoit vraiment criminelle, quand il s'agit de la santé \& de l'existence des hommes. » ${ }^{29}$ valeurs curiales et en déniant également au secrétaire perpétuel le pouvoir de juger d'un instrument quand l'impartialité dont il fait preuve est douteuse, il érige l'académie, dont il fait partie, en un tribunal qui vise au «bien de l'humanité » ${ }^{30}$. Selon lui, l'Académie doit rester l'instance experte, apte à juger de la valeur d'une invention ou d'une technique chirurgicale. Le " public ", quant à lui, peut toujours être trompé ; il est pour cela exclu de la sphère de légitimation. Ainsi en 1772, Valentin déplace encore une fois la question de l'évaluation des imprimés : pour lui, la diffusion du savoir chirurgical doit être soumise au contrôle de la communauté, comme instance collective égalitaire. Elle n'est plus, comme auparavant, sous la tutelle du roi et de son gouvernement, de la clientèle de l'imprimé ou encore des instances dirigeantes de l'Académie.

En 1791, Fourcroy qui commence sa nouvelle publication périodique, La Médecine éclairée par les sciences physiques, souligne l'ampleur des progrès chirurgicaux depuis la fin du siècle dernier, mais constate dans le même temps que, depuis vingt ans, la chirurgie

« s'est rallentie dans sa marche; les découvertes sur la nature et le traitement des maladies chirurgicales, les améliorations dans les opérations et dans les instrumens destinés à les pratiquer, après avoir été produites pour ainsi dire en foule par les Chirurgiens françois, se sont arrêtées tout-à-coup : la célèbre Académie de Paris n'a plus continué à répandre, par ses mémoires, les découvertes et les travaux de ses membres $»^{31}$

17 À la fin des années 1780, l'Académie Royale de Chirurgie semble bien être devenue une institution conservatrice. Après la suppression de l'apprentissage, elle a acquis un monopole sur l'éducation chirurgicale parisienne. Elle contrôle également étroitement le marché éditorial, aussi bien en centralisant les observations et les essais des chirurgiens du royaume, qu'en publiant le résultat de ses travaux dans ses Mémoires et en sanctionnant par son approbation la qualité des ouvrages imprimés. Lorsque ce modèle idéal de centralisation et de diffusion se grippe après 1775 , le contrôle que l'Académie exerce est de plus en plus mal supporté par ceux qui rejettent la censure du corps savant. 1789 bouleverse cet état de fait.

\section{La chirurgie et la liberté de la presse}

Lorsqu'en août 1789, les membres de la Constituante inscrivent la liberté de la presse au nombre des Droits de l'homme, les chirurgiens n'y voient pas d'emblée une aubaine. Leur pratique même justifie, pour Fourcroy, leur désaffection vis-à-vis de l'édition. Ce métier manuel laisse peu le loisir d'écrire à celui qui apporte des améliorations à sa technique, perdues à terme pour le monde savant ; « en un mot les Chirurgiens françois n'écrivent point assez " ${ }^{32}$. À la même date pourtant, certains praticiens expliquent différemment ce ralentissement de l'édition chirurgicale. Le Bruxellois Herbiniaux rapporte dans la seconde édition de son ouvrage les « pitoyables menées » de la censure parisienne. Arguant que son nouvel instrument pour la ligature des polypes de la matrice a reçu l'approbation de l'Académie Royale de Chirurgie, il avait justifié en 1782 la publication d'un second volume par la «nécessité imprévue de relever quelques erreurs de M. Baudelocque ", qui venait de faire paraître à Paris un ouvrage 
d'obstétrique encourageant l'utilisation de forceps ${ }^{33}$. Herbiniaux relate la mise sur le marché parisien de son ouvrage.

« Une nuée d'ennemis fondit tout-à-coup sur ma tête pour tâcher de faire proscrire mon ouvrage. Les uns sans entrer dans des discussions, qui auroient fait connoître leur ignorance sur la matiere que je traitois, employerent toutes les voyes possibles pour en empêcher le débit; les autres en firent un extrait qu'ils porterent à l'académie pour lui faire subir la censure la plus amère. [...] Cet ouvrage fut en effet arrêté à la chambre syndicale de Paris, \& M. le Bas, membre de l'Académie Royale de Chirurgie, nommé pour l'examiner, n'a pas jusqu'à présent voulu en faire son rapport à Mgr. le ci-devant Garde des Sceaux, quoique je n'aie cessé pendant plus de deux ans de le faire solliciter. ${ }^{34}$

Bien que l'Académie de Paris représente toujours l'autorité en matière de chirurgie, Herbiniaux se tourne vers d'autres institutions savantes, comme la Société Royale de Londres qui lui donne aussitôt son approbation. La proclamation de la liberté de la presse lui ouvre le vaste marché parisien.

Le chirurgien bruxellois met en cause le système de censure et les restrictions imposées à la publication chirurgicale, qui disparaissent en 1789. On observe en effet que l'édition spécialisée met plusieurs années à rattraper le retard accumulé. Dans le Journal de Fourcroy, plusieurs mémoires ont été communiqués à l'Académie Royale de Chirurgie. Le chirurgien-en-chef de l'Hôtel-Dieu de Rouen, Laumonier, publie par exemple en 1792, dans le dernier tome paru du Journal, ses "Observations sur le renversement et l'amputation de la matrice, lues à l'académie de chirurgie de Paris, le 9 décembre $1784 »{ }^{35}$. Raphaël-Bienvenu Sabatier, membre de l'Académie de Sciences, précise que ses remarques:

«ont été communiquées en différens temps à l'Académie de Chirurgie, lors des discussions qui se sont élevées dans son sein sur le traitement des fistules à l'anus, et n'ont plus rien de nouveau pour les membres de cette compagnie; mais comme elles manquent dans les traités de Pathologie et d'Opérations, ou qu'elles n'y sont pas exposées avec assez d'exactitude et de précision, et qu'elles peuvent être utiles, j'ai pensé qu'on me sauroit gré de les avoir rédigées et de les rendre publiques. » ${ }^{36}$

C'est bien la publication d'un savoir jusque là privé que l'académicien rend accessible au plus grand nombre. L'enceinte hermétique de l'Académie vient à disparaître, tandis que les communications qui y sont présentées sont aussitôt imprimées, comme l'» Observation sur une conception extra-utérine, lue à l'académie de chirurgie, par le citoyen Lacroix, ancien élève à l'école pratique de Paris $"{ }^{37}$.

D'autres attaques contre l'Académie de Chirurgie viennent également de l'hôpital. Le milieu hospitalier s'est substitué peu à peu au Collège de chirurgie pour la formation des étudiants. Xavier Bichat, l'élève et l'éditeur de Pierre-Joseph Desault, rapporte de manière circonstanciée la carrière du futur chirurgien-en-chef de l'Hôtel-Dieu. Sans le "talent, qui assure [le] succès dans les sociétés", Desault fut nommé au comité perpétuel de l'Académie Royale de Chirurgie; il y a présenté un mémoire et s'en est rapidement désintéressé. Pour son élève, il ne pouvait y réussir et publier « ces dehors toujours brillans, quelquefois stériles, sur lesquels [Antoine] Louis établit en partie les bases de sa réputation " ${ }^{38}$. C'est à l'hôpital, d'abord à la Charité, puis à l'Hôtel-Dieu, que Desault connut un succès peu commun, amplifié en janvier 1788 par le réaménagement de l'amphithéâtre dans le bâtiment méridional, depuis longtemps réclamé par le chirurgien-en-chef ${ }^{39}$. Pour Bichat,

«[les recherches] de Desault n'avoient encore été publiées que par la foule des

élèves qui se pressoient pour l'entendre; souvent en passant de bouche en bouche, 
elles arrivoient défigurées dans le monde savant, \& l'empressement à les recevoir, en les faisant plus vite circuler, multiplioit les erreurs. Il entreprit, pour les éviter un ouvrage périodique, où l'exposé de sa doctrine, confirmé par les résultats de sa pratique, présenta une suite de faits \& de préceptes, qui méthodiquement rassemblées devoient former un jour les bases d'un traité plus complet. " ${ }^{40}$

Desault établit en 1791 un journal dont « on prend souscription à l'Hôtel-Dieu, ou au 151, rue de la Harpe ». Il organise ainsi une véritable entreprise de presse, dont les rédacteurs sont les chirurgiens et les étudiants de l'Hôtel-Dieu, qui constituent sans doute également une grande part de sa clientèle. Dans le Journal de chirurgie, on retrouve le phénomène de rattrapage évoqué plus haut: il fait ainsi paraître un «Extrait d'un Mémoire de M. Desault sur la Luxation de l'extrémité inférieur du Radius, lu dans la séance publique de l'Académie de chirurgie, en 1777, et non imprimé $\$\left[20{ }^{[11}\right.$. D'emblée, le projet de Desault se veut concurrent des publications académiques. L'Académie royale de chirurgie est accusée implicitement de "lèse-humanité ", puisqu'elle ne communique ni ses découvertes ni ses observations, au risque de causer la mort de plusieurs individus ${ }^{42}$.

Pour Desault, l'imprimé permet d'abord d'assurer la parole enseignée et d'établir fermement le savoir chirurgical. En un sens, un tel transfert de l'autorité du savoir chirurgical, des Mémoires au Journal, manifeste la redistribution des pouvoirs de l'Académie à l'hôpital ${ }^{43}$. Par ailleurs, la forme périodique constitue le «moyen de communication le plus prompt, le plus certain et le moins dispendieux ; [... elle] offre d'ailleurs tant d'avantages, qu'on a lieu de s'étonner qu'on n'en ait conçu l'idée qu'après le milieu du XVIIIe siècle ». Le projet éditorial est ainsi conçu dans sa totalité sociale. Peu coûteux, il s'adresse aussi bien à l'étudiant qu'au praticien confirmé ; économe de ses mots, il omet de rapporter la tradition d'un traitement pour ne garder que l'actualité de l'observation ou la nouveauté de la technique ; il est la

«propriété de tous ceux qui concourent par leur travaux à sa perfection; en publiant leur nom avec leurs productions, il rapporte à chacun le tribut de gloire qui lui est dû ; il offre un moyen de correspondance facile entre tous les gens de l'art, quelques pays qu'ils puissent habiter ; il ouvre un champ libre à la discussion, souvent nécessaire, pour dissiper des incertitudes et mettre une vérité dans tout son jour. ${ }^{44}$

Le Journal de Desault doit constituer ainsi un espace de discussion dont la publicité et la diffusion à l'échelle de l'Europe. Cette entreprise audacieuse repose sur la réputation internationale de Desault, qui attire auprès de lui de nombreux étudiants. Parmi les rédacteurs, on trouve G. Reumont et $\mathrm{M}$. Brewer, docteur en médecine, qui traduisent des extraits récents de travaux allemands ${ }^{45}$. La plupart des journalistes-étudiants retranscrivent les leçons sur les maladies urinaires que Desault donnait dans l'amphithéâtre, ou encore les observations qu'il faisait à l'hôpital ou dans ses visites privées. Le Journal de Chirurgie en vient à remplacer les Mémoires de l'Académie jugés défaillants; il publie les annonces de prix, l'éloge, anonyme et caustique, d'Antoine Louis, les pétitions sur les patentes et les extraits de registres du corps savant.

Le Journal de Chirurgie est salué partout comme une entreprise remarquable. Il est rapidement traduit à Londres et son contenu est largement repris par les autres journaux médicaux ; Noël Retz, dans ses Nouvelles ou Annales de l'art de guérir consacre le « chirurgien éclairé sur l'étendue et l'importance de son art » et l'innovation éditoriale ${ }^{46}$. Pour notre propos, les ouvrages périodiques sont essentiels : ils permettent d'étudier la redéfinition du public qui s'opère pendant les premières années de la Révolution. Le 
rédacteur de périodique Retz, "médecin du roi servant par quartiers", auquel il s'ajoute le titre de «médecin des hôpitaux de la marine pendant la dernière guerre " à partir de 1790, est particulièrement soucieux d'élargir le cercle de ceux qu'il souhaite instruire. La même sollicitation apparait dans la liste de ses œuvres, signalées avec leur prix, au début du journal. S'inscrivant en partie dans la lignée de la Gazette de santé, il prend le parti de d'informer le public sur les erreurs et les remèdes dangereux. Il cherche surtout à toucher « les Gens du monde qui ont pris depuis quelque temps du goût pour les connoissances médicales, \& qui trouvoient la peine de les puiser dans une quantité de livres, rebutante ", grâce à un digest commode des ouvrages parus dans l'année ${ }^{47}$. C'est pourtant le seul à opter pour cette « destination problématique faite de l'association de deux publics incompatibles ${ }^{48}$. Les autres périodiques s'adressent plus exclusivement au public médical. L'un d'entre eux même, le Journal de Médecine, Chirurgie et Pharmacie, \&c., se présente comme le moyen d'instruire les «médecins et chirurgiens du royaume », caractérisés en partie par leur " peu de fortune »; en janvier 1790, il réussit à obtenir de l'administration la franchise de port pour les départements, et dessine un plan plus général de formation, où « le département se chargera de payer annuellement la somme de cent livres " pour couvrir les frais d'une telle entreprise ${ }^{49}$.

L'aventure du Journal de Chirurgie se clôt avec le quatrième tome dans lequel Bichat publie un éloge de Desault, son maître. Pour l'élève, « les circonstances interrompirent ce journal, \& la mort l'empêcha d'en reprendre le travail ${ }^{50}$. Bien que les comptes du journal ne soient pas aujourd'hui conservés, on peut comparer la situation que le Journal de Desault connaît à la fin de 1792 avec celle du journal de Fourcroy, La Médecine éclairée par les sciences physiques. Le 15 décembre 1792, Fourcroy publie un «Avis aux Souscripteurs de la Médecine éclairée » selon lequel « les circonstances ne permettant point aux auteurs de ce journal de le continuer sous la même forme, il ne paroîtra plus tous les quinze jours; et il n'y aura plus de souscriptions " ${ }^{51}$. C'est ainsi que prend fin une époque de liberté pour la chirurgie, au cours de laquelle elle a ouvert un marché nouveau pour les formes brèves et périodiques, formes particulièrement bien adaptées à une communication rapide des observations. Avant même la disparition des sociétés royales, la discipline chirurgicale a quitté l'enceinte de l'Académie de Chirurgie pour se diffuser largement auprès des nombreux étudiants des hôpitaux et auprès de tous les chirurgiens du royaume qui souhaitent accéder à ce savoir. La "République de la chirurgie » est née.

III. Nouveaux auteurs, nouveaux publics? La chirurgie sous le patronage de l'État, 1793-1799

$\mathrm{Au}$ cours du premier semestre 1793, la diffusion de la chirurgie se ralentit considérablement. Ce phénomène traduit partiellement la longue agonie que connait l'Académie royale de Chirurgie à ce moment. Dès mars, le Comité d'Instruction Publique supprime les rentes qui finançaient les collèges ${ }^{52}$; en dépit de vives résistances de la part des chirurgiens pour surseoir à une décision inévitable, le décret du 8 août 1793 qui abolit les sociétés savantes supprime une Académie exsangue ${ }^{53}$. Concourent à l'étiolement de l'activité de l'institution à partir de 1792, les enrôlements, que de nombreux documents attestent, et surtout, la désagrégation de la bibliothèque du Collège de chirurgie ${ }^{54}$. Cette collection, très fournie par le legs initial de La Peyronie, avait été développée par les soins constants du bibliothécaire Bernard Peyrilhe, puis d'Antoine Louis. Celui-ci avait obtenu l'autorisation expresse d'entreposer ses propres ouvrages avec ceux du Collège, en échange de la promesse d'en faire donation à sa mort. Le deuxième testament de Louis en 1792 revient sur ces dispositions. À la mort du 
secrétaire perpétuel, il est violemment contesté par les chirurgiens, qui demandent justice, sans succès ${ }^{55}$. En la personne de Pierre Süe, premier bibliothécaire de l'École de Santé, ce sont les chirurgiens qui réorganiseront le fonds lors de la refonte des institutions médicales.

Les années 1793-1794 marquent un coup d'arrêt des publications chirurgicales : seules cinq monographies de chirurgie voient le jour entre le 1er janvier 1793 et le 21 septembre 1794, à comparer avec les douze parues en $1792{ }^{56}$. C'est même l'ensemble de l'industrie du livre qui connaît une crise sans précédent, comme l'a bien montré Carla Hesse ${ }^{57}$. L'importante inflation de l'an II contribue au marasme de l'édition et affecte particulièrement toutes les entreprises éditoriales à souscription. Les journaux médicaux, publiés entre 1789 et 1791, disparaissent à la fin de 1792; le Journal de médecine, chirurgie, pharmacie, \&c. édité par Bacher cesse de paraître au cours du premier semestre 1793. Les quelques ouvrages de chirurgie qui paraissent sont surtout des rééditions.

À partir de 1795, deux facteurs contribuent au renouvellement de la diffusion chirurgicale après ce long silence: la refonte des institutions de médecine et les subventions de l'État aux ouvrages scientifiques. Le décret du 14 frimaire an III avait institué les trois Écoles de santé de Paris, Strasbourg et Montpellier, qui remplacent les cadres de l'Ancien Régime; parmi les premières publications de l'an III, on trouve un "Avis sur la continuation des Mémoires de la Faculté de Médecine, de l'Académie de Chirurgie et de la Société de médecine, publié par les Professeurs de l'École de Santé de Paris ", revêtu de la signature de Thouret, directeur. Selon cet Avis :

«chargée spécialement par l'article VII du décret du 14 frimaire, an 3, qui l'a établie, de concourir à l'amélioration de l'art, [l'École de santé] a compté au nombre de ses plus utiles travaux de recueillir avec soin les mémoires et les observations déposés dans les archives des diverses Compagnies médicales supprimées en août 1793. $»^{58}$

31 L'Avis prévoit ainsi de "livrer à l'impression la suite et la fin de la collection, sous la double forme de Prix et de Mémoires » de l'Académie de Chirurgie ${ }^{59}$. L'École de Santé se donne pour mission de publier les travaux, restés dans les archives de la Faculté de Médecine, de l'Académie de Chirurgie et de la Société Royale de Médecine qu'elle a reçues en héritage. Des Mémoires et des Prix de l'Académie de Chirurgie, seuls les seconds paraissent, en 1798, an VI, chez Didot le jeune ${ }^{60}$. Pourtant une alliance est nouée entre l'École de santé et l'État, sur le même motif d'utilité publique qui avait justifié le monopole de l'Académie de Chirurgie. En effet, les sujets abordés en priorité sont ceux des maladies des os et des plaies d'armes à feu et «tout ce qui tient à la santé des défenseurs de la patrie, inspire, en ce moment sur-tout, un vif intérêt aux amis de l'humanité ${ }^{61}$.

Le second facteur propice à la rénovation de la publication médicale - Carla Hesse l'a parfaitement démontré - réside dans la politique de soutien à la publication scientifique 62. S'il reste à montrer qu'elle favorise la chirurgie, plusieurs indices le laissent supposer : Didot l'aîné, connu pour les subventions qu'il a obtenues pour l'impression d'ouvrages à caractère scientifique, fait imprimer le premier manuel de RaphaëlBienvenu Sabatier, «chirurgien en chef à la maison nationale des Invalides, Professeur à l'École de Santé, et membre de l'Institut National ». En faisant paraître cet ouvrage, Sabatier dit remplir «l'engagement (...) contracté depuis longtemps, de publier les cahiers qui ont servi de base aux leçons [qu'il donnait] au Collège de Chirurgie sur l'art d'opérer, et [qu'il] donne encore à l'École de santé sur la médecine opératoire ». Il 
ajoute qu' "en cédant à l'empressement de ceux qui ont désiré que [il] le [prît], [il a] moins consulté [ses] forces que l'envie de [se] rendre utile " ${ }^{63}$. Son ouvrage paraît peu après celui de Pierre Lassus, professeur de pathologie externe à l'École de santé, paru chez Buisson et portant le même titre. Bien que Sabatier ne désigne pas ceux qui l'ont pressé d'imprimer son ouvrage, on peut penser que l'initiative est venue à la fois de l'éditeur et des pouvoirs publics. La production chirurgicale entre l'an III et l'an VII est surtout représentée par les traités issus des cours de l'École de santé et la chirurgie des armées. Trois éditeurs se partagent le marché de l'édition chirurgicale dans les dernières années du siècle: Pierre Didot le jeune, Théophile Barrois et André Croullebois éditent également des monographies et les nouveaux recueils périodiques qui apparaissent avec la restructuration de la communauté.

$\mathrm{Au}$ vu des auteurs qui publient sous le Directoire, on ne peut parler de rupture révolutionnaire, mais plutôt de monopole accru des institutions gouvernementales. Pourtant, outre les professeurs de l'École de santé, on voit émerger des auteurs nouveaux, les sociétés médicales qui apparaissent à partir de l'an IV ${ }^{64}$. Elles revendiquent le statut collectif des journaux qu'elles publient. La Société de Santé, qui devient rapidement Société de Médecine, tient sa première séance le 2 germinal an VI (22 mars 1796), sous les auspices du gouvernement ${ }^{65}$. Elle se donne pour objectif de faire imprimer le travail résultant du concours des praticiens de "plusieurs branches de la Médecine, qui ne dûrent la rapidité de leur accroissement en France qu'à la réunion des Maîtres de l'Art», et qui "languissoient depuis la destitution des Compagnies savantes " ${ }^{66}$. Créée pour " pour l'instruction mutuelle de ses membres, ses travaux portent sur l'art de guérir, et les sciences qui peuvent l'éclairer ", la Société Médicale d'Émulation publie également des mémoires ${ }^{67}$. Les journaux médicaux inventés par la Révolution évoluent ainsi quelque peu. Les journaux de 1789 étaient l'œuvre d'un rédacteur unique, comme les Nouvelles de Retz, ou d'un éditeur pour lesquels différents scripteurs oeuvraient, comme le Journal de Desault. Les périodiques de l'an $\mathrm{V}$ représentent des résultats obtenus collectivement : ils émanent de sociétés qui établissent une définition d'un savoir orthodoxe de la médecine, à l'aune des normes établies par le corps professoral. Ces sociétés restructurent le marché périodique spécialisé sous le patronage du gouvernement, qui fournit les locaux de réunion et certainement une partie des fonds pour l'impression. En raison de l'approbation gouvernementale à laquelle elles sont soumises, ces sociétés structurent en profondeur la communauté médicale, tout en étant travaillées par les rapports de forces qui y règnent. Ainsi l'appartenance des membres souligne la prééminence de l'hôpital et de l'École de Santé sur la communauté médicale ${ }^{68}$.

La diffusion de la chirurgie après l'an IV définit de manière nouvelle le public auquel elle se destine. En un sens, on assiste à une relative fermeture de l'offre chirurgicale sur le groupe médical lui-même. Plus précisément, celle-ci organise un système de diffusion qui vise l'ensemble de la communauté, en commençant par les étudiants. Avant 1789, plusieurs parutions, marginales cependant, manifestent cette volonté de diffuser le savoir à tous les groupes médicaux. Il y a certes les Principes de chirurgie de La Faye, réédités à dix reprises entre 1746 et 1797, mais cet exemple montre plutôt le besoin criant de manuels. Plusieurs projets d'offrir aux sages-femmes les rudiments de l'obstétrique ont également vu le jour ${ }^{69}$. Le mouvement a continué au début de la Révolution, à destination plus généralement des médecins et des chirurgiens de campagne, comme on l'a vu avec le Journal de Médecine. Noël Retz constitue un bon exemple de cette évolution. Lorsque son périodique annuel, les Nouvelles, ou annales de 
l'art de guérir, destiné aux « Gens du monde », disparaît en 1792, il entrevoit l'émergence d'une nouvelle clientèle, pour qui il publie à compte d'auteur un petit opus en l'an II, son Instruction aux jeunes chirurgiens sur les fièvres les plus communes dans les armées de la République française. Les chirurgiens des armées, enrôlés sans formation véritable, deviennent une clientèle privilégiée de la chirurgie.

Bientôt, cependant, ce sont les étudiants de médecine qui sont plus particulièrement courtisés. Dans son Tableau de Paris, Mercier avait repéré la figure montante de ce débutant : il y peint l'étudiant en chirurgie à l'ombre d'une muraille, qui attend l'issue malheureuse d'un duel pour emporter le cadavre ou qui découpe, dans sa chambre mal éclairée, des corps volés au cimetière ${ }^{70}$. En 1793, Deschamps est l'un des premiers à justifier la réédition à compte d'auteur d'un article du Journal de Fourcroy, portant sur la méthode de ligature de l'artère poplitée de John Hunter ${ }^{71}$. À partir de l'an IV, les étudiants deviennent les destinataires privilégiés des ouvrages publiés. C'est le cas du Traité des bandages et appareils de Thillaye. «Cet ouvrage étant destiné particulièrement aux élèves qui sont employés à l'armée, [il a] cru devoir entrer dans de longs détails sur les préceptes de cette branche de l'art de guérir " ${ }^{72}$. La vente de son ouvrage se fait d'ailleurs «à Paris, chez l'Auteur, rue et Ecole de médecine, [chez] le Portier de ladite École [et] Viller, Libraire, rue des Mathurins, n 396 ", comme l'annonce la page de titre. Ces ouvrages à destination des étudiants de médecine et de chirurgie se multiplient. En effet, dans les Écoles de santé, devenues à partir de l'an XI Facultés de médecine, les élèves constituent un public nouveau, éduqué et stable. Ce marché est essentiellement contrôlé par les enseignants de l'École de Santé, comme on peut le vérifier en étudiant les inventaires après décès des chirurgiens morts entre 1810 et 1834 . Une étude de vingt-deux inventaires après décès retrouvés montre que les petites collections des chirurgiens contiennent en très grande majorité des ouvrages des grands professeurs parisiens ${ }^{73}$. La diffusion de la chirurgie pendant les années révolutionnaires se resserre donc sur la communauté médicale, celle-ci étant pensée comme l'union de groupes différents dont il faut homogénéiser le savoir. Toute tentative de contourner cette mainmise, comme on le voit dans la polémique anti-césarienne, se solde par un échec ${ }^{74}$.

La Révolution française n'a pas constitué une rupture dans la diffusion des savoirs médicaux. Au contraire, les dernières années du XVIIIe siècle concluent la lente émergence d'un monopole des institutions médicales sur le marché de l'imprimé spécialisé et consacrent le livre comme vecteur essentiel de l'information. Si l'on considère le cas particulier de la chirurgie, le contrôle a commencé dès les années 1760 , où la direction de la Librairie a confié la censure des ouvrages à l'Académie Royale de chirurgie. Le public, à qui était laissé jusque là la sanction du succès d'une technique ou d'un ouvrage, est alors disqualifié, ; il est dès ce moment jugé trop souvent victime d'auteurs hétérodoxes, de « charlatans ».

Pourtant l'an I de la Liberté est bien vécu comme une révolution par les chirurgiens pénalisés par le silence de l'Académie à partir de 1775. Dès lors on observe un phénomène de rattrapage de la publication chirurgicale et des innovations, dont la plus importante est le Journal de Chirurgie de Desault. Les périodiques montrent en 1790 la relative hétérogénéité des destinataires de la chirurgie. Ils amorcent la redéfinition de la politique de communication médicale, nettement affirmée après la crise éditoriale de l'an II. Placée sous le puissant contrôle de l'École de Santé, nouvellement fondée et héritière des institutions de l'Ancien Régime, la diffusion qui s'organise s'appuie sur le réseau des sociétés de médecine et vise la communauté médicale dans son ensemble, en 
s'adressant successivement aux médecins et chirurgiens des départements, de l'armée, et bientôt aux étudiants. Les formes de la vulgarisation de la fin du XVIIIe siècle traduisent paradoxalement la réinvention d'un corporatisme médical.

* Cet article est issu d'une thèse, «L'invention du geste chirurgical, France et GrandeBretagne, 1760-1830 ", préparée sous la direction de Pietro Corsi, à l'Université de Paris 1. Je remercie vivement mes relecteurs patients, Julien Aliquot, Katia Béguin, Manuel Charpy, Natalia Muchnik et Benoist Pierre, pour leurs remarques judicieuses. Pour les citations des textes imprimés et manuscrits, j'ai respecté l'orthographe originale.

\section{NOTES}

1.Gazette de Santé contenant les nouvelles découvertes sur les moyens de se bien porter et de se guérir quand on est malade, n52, déc. 1789, p. 205.

2.Pierre RETAT, Journaux de 1789. Bibliographie critique, Paris, CNRS, 1988, pp. 282-284.

3.Gazette de Santé (1773), Préface, np.; Journal de Médecine, Chirurgie, Pharmacie, etc., t. 82 (janvier 1790), p. 21.

4.Voir par exemple, Roy PORTER, Health for sale. Quackery in England, 1660-1850, Manchester, Manchester University Press, 1989 ; Anne DIGBY, Making a medical living. Doctors and patients in the English market for medicine, 1720-1911, Cambridge, Cambridge University Press, 1994.

5.Cf. l'introduction de ce numéro, Jean-Luc CHAPPEY, « Enjeux sociaux et politiques de la "vulgarisation scientifique" en Révolution (1780-1810».

6.En l'absence d'études synthétiques sur la chirurgie française, l'étude de Toby GELFAND, Professionalizing Modern Medicine. Paris Surgeons and Medical Science and Institutions in the 18th Century, Wesport (Conn.), Greenwood Press, 1981, reste très utile.

7.François tolet, Traité de la lithotomie ou de l'extraction de la pierre hors la vessie, Paris, chez l'autheur, 1682, fig. p. 140 ; St. EVERS, « Tableau de l'opération de la taille, von Marin Marais (1725) : eine Steinschnittoperation in Musik dargestellt », Urologe, Ausgabe A, 32/3 (May 1993), pp. 254-259.

8.Antoine LILTI, « Le monde des salons. La sociabilité mondaine à Paris dans la seconde moitié du XVIIIe siècle ", Thèse d'histoire, Université Paris I, 2003, cf. particulièrement le chap. 8 « La société de spectacle ». Citation p. 538.

9.Mathew RAMSEY, Professional and popular medicine in France, 1770-1830: the social world of medical practice, Cambridge, Cambridge University Press, 1988 ; pour une étude récente sur le phénomène à Londres au 17e siècle, cf. Margaret PELLING, Medical conflicts in early modern London : patronage, physicians, and irregular practitioners 1550-1640, Oxford, Oxford University Press, 2003.

10.Archives Nationales (A.N.) Ét XCII/916. 19 août 1786.

11.Journal historique sur les matières du temps, novembre 1748, pp. 383-391. Jean-Pierre KUNNERT, dans « La Clef du Cabinet de Princes, 1704-1773 » in Jean SGARD (dir.), Dictionnaire des journaux, 1600-1789, Paris, 1991, p. 234, confirme les chiffres avancés par 
le journal lui-même par le nombre très important des collections de ce périodique qui sont conservés en Europe.

12.Académie Nationale de Médecine, fonds Académie Royale de Chirurgie (A.R.C.), carton 8 , dossier 7 , relatif aux placards de Villiers, pièce 2 « Lettre de Villiers à Morand » (21 février 1762).

13.Jean EHRARD et Jacques LEONARD, « Deux périodiques français du 18e siècle : “le Journal des savants" et "les Mémoires de Trévoux" ", in François FURET, Daniel ROCHE, Jacques Roger (dir.) Livre et société dans la France du XVIIIe siècle, Paris, 1965, pp. 33-59. 14.Philip wiLson, « An Enlightenment Science? Surgery and the Royal Society » in Roy PORTER (dir.), Medicine in the Enlightenment, Amsterdam, 1995, pp. 360-386.

15.Cité par David A. KRONICK, «Medical "Publishing Societies" in eighteenth-century Britain », Bulletin of the Medical Library Association, 1994, 82 (3), p. 280.

16.Mémoires de l'Académie Royale de Chirurgie. Paris, t. I, C. Osmont, 1743 ; t. II, Delaguette, 1753 ; t. III, Veuve Delaguette, 1757 ; t. IV, P.-Alex. Le Prieur, 1758 ; t. V, Didot.

17.MARJolin (dir.), Encyclopédie des Sciences Médicales ou Traité général, méthodique et complet des diverses branches de l'art de guérir. Septième division : Collection des ouvrages classiques. Mémoires de l'Académie royale de chirurgie, précédés d'une analyse de ces mémoires par M. Le professeur Marjolin et suivis de deux mémoires inédits, Paris, Bureau de l'Encyclopédie, 1836, p. 1.

18.Rééd. P.-Alex Le Prieur, in-12, 1764-1774; Memoirs of the Royal Academy of Surgery at Paris, containing a great variety of cases, in the chief branches of the art. Many of them very surprising and uncommon. Translated from the orginal dedicated to the French King. 2 vol. London, E. Cave, 1750 ; 2e éd., 3 vol., J. Rivington and J. Fletcher, 1759.

19.Article « Chirurgie », in DIDEROT \& D'ALEMBERT (dir.), Encyclopédie ou Dictionnaire raisonné des sciences, des arts et des métiers t. III, Paris, Briasson, David l'aîné, Le Breton, Durand, 1753, pp. 350-355.

20.François PAUL (dir.), Dictionnaire de chirurgie, communiqué à l'Encyclopédie par M. Louis. Paris, Saillant \& Nyon, 1772 ; DE LA ROCHE \& PETIT-RADEL, Encyclopédie méthodique. Chirurgie, Paris, Panckoucke, 1792, 3 vols.

21.A.R.C., carton 8 , dossier 7 , pièce 3 , « Coppie de la lettre écrite de la part de l'academie a M. de Villiers, chirurgien au Mans le 12 mars $1762 »$.

22.A.R.C., carton 11B dossier 78 « La Martinière à Morand. Lettre à propos de la création d'un Journal de chirurgie à laquelle il est opposé (1761) ».

23.A.R.C. carton 11A, dossier 40 « Pibrac présente un mémoire à Lamoignon pour faire retirer les privilèges accordés à la Compagnie des libraires pour un Journal de chirurgie (1766)».

24.A.R.C. carton 8, dossier 16, pièces 4, « Pibrac à Morand. Lettre écrite à Morand pour lui remettre la clé de l'armoire où sont enfermés les titres et les papiers de l'Académie (1763, 18 juin) »; 8, « Pibrac à Morand. Lettre où Pibrac demande à Morand d'appliquer des règles plus strictes pour la tenue des différents registres » et 10, «Pibrac. Extrait du règlement de l'Académie des sciences concernant les papiers et les registres. Extrait de l'Histoire de l'Académie des belles-lettres (t. 27) avec substitution d'un secrétaire perpétuel à un autre ".

25.A.R.C., carton 11B dossier carton 11A, dossier 17 « Demande de Serain d'approbation par l'A.R.C. d'un "plan d'un nouvel ouvrage périodique" et réponse de Louis » (1775). 26.Abbé DINOUARD, Abrégé de l'embryologie sacrée ou Traité du devoir des Prêtres, des médecins, des chirurgiens \& des sages-femmes envers les enfans qui sont dans le sein de leur 
mère, 2e édition, Paris, Nyon, 1766, à comparer avec Id., Abrégé de l'embryologie sacrée ou Traité du devoir des Prêtres, des médecins \& autres, sur le salut éternel des enfans qui sont dans le ventre de leur mère, Paris, Nyon, 1762.

27.Cf. par exemple The London Medical Journal, by a Society of Physicians. vol. 2. London, 1782, « Preface », p. III.

28. [Louis-Antoine] VALENTIN, Recherches critiques sur la chirurgie moderne. Avec des Lettres à M. Louis, Docteur en Droit, Professeur, Docteur en Chirurgie, Chirurgien consultant des Armées du Roi, Secretaire perpétuel de l'Académie royale de Chirurgie, \&c., Amsterdam [Paris], Gogué, 1772.

29.Ibid., p. 22.

30.Ibid., p. 24.

31.[Antoine FOURCROY], La Médecine éclairée par les sciences physiques ou Journal des Découvertes relatives aux différentes parties de l'art de guérir. Paris, Buisson, 1791, t. I, p. 38. 32.Ibid.

33.G. HERBINIAUX, Traité sur divers Accouchemens laborieux et sur les polypes de la matrice. Ouvrage dans lequel on trouve la description d'un nouveau Levier, imité de celui de Roonhuysen, \& mis en parallele avec le forceps : ainsi que d'un nouvel instrument, propre à la ligature des Polypes, approuvé par l'Académie Royale de Chirurgie de Paris, vol. 2, Bruxelles, J. L. de Boubers, 1782, p. II. Cf. aussi Jean-Louis BAUDELOCQUE, L'art des accouchemens, Paris, Méquignon l'aîné, 1781. Sur le débat concernant l'utilisation de forceps, voir Adrian WILSON, The making of man-midwife. Childbirth in England 1660-1770, Londres, UCL Press, 1995.

34.G. HERBINIAUX, op. cit., 2e éd., Bruxelles, Lemaire, 1791, pp. IV-V.

35.Antoine FOURCROY, op. cit., t. I, pp. 33-48.

36. Antoine FOURCROY, op. cit., t. II, p. 206.

37. Antoine FOURCROY, op. cit., t. IV, pp. 346-42.

38.Xavier віснат (éd.), Euvres chirurgicales de P. J. Desault, chirurgien en Chef du grand Hospice d'Humanité, ci-devant Hôtel-Dieu de Paris ou Tableau de sa Doctrine et de sa Pratique dans le Traitement des Maladies externes. Ouvrage publié par Xav. Bichat, son Élève, Paris, Veuve Desault, Méquignon l'aîné, Devilliers, Deroi, 1798, pp. 30-31.

39.Archives de l'Assistance publique, liasse 1438, « Registre des délibérations du Bureau de l'Hôtel-Dieu, pour l'année 1787 », 21 novembre 1787, F685, cité par Marie-Christine LORANG, Les tentatives de réforme de l'Hôtel-Dieu de Paris à la fin du XVIIIe siècle, Paris, Positions des thèses de l'École des Chartes, 1975, p. 361.

40.Xavier BICHAT, op. cit., p. 29.

41.Journal de chirurgie, vol. 1, 1791, p. 78 sq.

42.Ibid., p. 4.

43.Toby GELFAND, op. cit., pp. 118-119.

44.Journal de chirurgie, vol. 1, 1791, p. 6.

45.Ibid., t. 1, « Extrait du Journal de médecine de Francfort, Année 1789, IIIe partie, p. 134 et suiv. traduit de l'allemand, par M. Brewer, docteur en médecine », pp. 264-268 et t. 3, « Extrait des mélanges de Chirurgie de Schmucker, traduits de l'allemand par G. Reumont ", pp. 57-61.

46.Parisian Chirurgical Journal, by Monsieur Desault, principal surgeon to the Hotel-Dieu, Paris. Translated into English by R. Gosling, Surgeon, London, the translator and M. Boosey, 1793 ; [Noël RETz], Nouvelles, ou Annales de Médecine, Chirurgie \& Pharmacie. Recueil raisonné de 
Tout ce qu'il importe d'apprendre pour être au courant des connoissances \& à l'abri des erreurs, relatives à l'Art de guérir, t. VI, Paris, Méquignon l'aîné, 1791, p. 384.

47.Nouvelles..., « Dédié à S.A.S. Monsieur le Duc d'Orléans, premier Prince de Sang ", t. V, 1789. « Avertissement », p. 6.

48. Claire CAZANAVE, « Une publication invente son public. Les Entretiens sur la pluralité des mondes » in Christian JUHAUD, Alain VIALA (dir.), De la publication. Entre Renaissance et Lumières, Paris, Fayard, 2002, pp. 267-280, citation p. 280.

49. "Second Mémoire sur la Médecine. Moyen de Perfectionner l'art de guérrir », Journal de Médecine, Chirurgie, Pharmacie, \&c. t. 82, Paris, Imprimerie de Monsieur, janvier 1790, pp. 5-23, citations pp. 15 et 21.

50.Xavier BICHAT, op. cit., p. 31.

51.Antoine FOURCROY, op. cit., t. IV, p. 321.

52.M. J. guillaume (dir.), Procès-verbaux du Comité d'Instruction Publique, t. II, 3 juillet 1793-30 brumaire an II (20 novembre 1793), Paris, Imprimerie Nationale, 1894, pp. 308-309.

53.M. J. guillaume (dir.), op. cit., p. 249.

54.Décret de la Convention Nationale qui met à la réquisition du ministre de la guerre les officiers de santé, Paris, Imprimerie Nationale, an II. Parmi les témoignages d'enrôlement, cf. par exemple, Bertrand LAGRESIE, Observations sur l'amputation de la cuisse nécessitée par la spina ventosa du tibia, Paris, Migneret, an VII, p. 5 sq.

55.A.R.C. carton 60, dossier 2, pièce 195. La collection de Louis est rapidement vendue, le 7 janvier 1793, par le libraire Née de la Rochelle, d'après le catalogue de vente publié à l'occasion.

56. Ces décomptes sont effectués à partir des catalogues de bibliothèques (BIUM et Wellcome Library).

57. Carla HESSE, Publishing and Cultural Politics in Revolutionary Paris, Berkeley, University of California Press, 1991, p. 126 sq.

58.Avis sur la continuation des Mémoires de la Faculté de Médecine, de l'Académie de Chirurgie et de la Société de médecine : publiée par les Professeurs de l'Ecole de Santé de Paris, Paris, Imprimerie de la République, s. d. [1794], p. 1.

59.Ibid.

60.Mémoires sur les sujets proposés pour les prix de l'Académie de Chirurgie. Tome V. De l'année 1775 , jusques et compris 1783, Paris, Didot le jeune, an VI.

61.Avis..., op. cit., p. 2.

62.Carla HESSE, op. cit., p. 183 sq.

63.[Raphaël-Bienvenu] SABATIER, De la Médecine opératoire, ou des opérations de chirurgie qui se pratiquent le plus fréquemment, Paris, Didot le jeune, 1796.

64.Elles restent aujourd'hui très mal connues. Cf. Pierre HUARD, Marie-José IMBAULTHUART, «Les sociétés d'étudiants en médecine au début du XIXe siècle », 95e Congrès National des Sociétés Savantes (Reims, 1970), t. 2, Paris, 1975, pp. 229-238 ; Florent PALLUAULT, Étudiants et praticiens au service de la médecine : la Société anatomique de Paris de 1803 à 1873. Étude institutionnelle et prosopographique, Mémoire de DEA Archivistepaléographe, École des Chartes, 1999.

65. Recueil périodique de la Société de Santé, t. 1, Paris, Barrois et Croullebois, vendémiaire, an V (Octobre 1796), « Avant-propos », p. 3.

66.Ibid., Prospectus. 
67. "Notice de la Société médicale d'émulation », Magasin Encyclopédique, ou Journal des Sciences, des Lettres et des Arts, t. III, p. 260.

68.Pour une étude plus générale, cf. Jean-Luc CHAPPEY, « Les sociétés savantes à l'époque consulaire », A.H.R.F., 309/3 (1997), pp. 451-462, dont je reprends ici une partie des analyses.

69.Anne Amable AUGIER DU FOT, Catéchisme sur l'art des accouchemens pour les sages-femmes de la campagne, fait par l'ordre et aux dépens du gouvernement. Soissons, "Chez les libraires », 1775 ; Montpellier, Jean Martel aîné, 1776 ; Paris, Didot le jeune \& Ruault, 1784 .

70.Louis-Sébastier MERCIER, « Jeunes chirurgiens », Tableau de Paris II (1e éd., 1782), éd. établie sd. Jean-Claude BONNET, Paris, Mercure de France, 1994, pp. 645-646.

71.[Jean François Louis] DESCHAMPS, Observations sur la ligature des principales artères des extrémités, à la suite de leurs blessures, et dans les anévrismes, particulièrement dans celui de l'artère poplitée, dont deux ont été opérés suivant la méthode de Jean Hunter, Chirurgien anglais. Observations qu'il a insérées dans le journal du citoyen Fourcroy, tome III, $n^{\circ} \mathrm{III}$, et tome I, $n^{\circ} \mathrm{XI}$, Paris, Claudé, 1793.

72.[Jean Baptiste Jacques] THILlAYE, Traité des Bandages et des Appareils, Paris, Viller, an VI (1798), p. VIII.

73.Étude en cours, à paraître dans la thèse " L’invention du geste chirurgical ». 74.Bernard THIs, La requête des enfants à naître, Paris, Le Seuil, 1982.

\section{RÉSUMÉS}

Ce travail propose une réflexion sur la vulgarisation et la diffusion des savoirs médicaux, à partir de l'exemple de la chirurgie. Étudiant les structures institutionnelles et économiques de la diffusion, il aborde la question de la construction des destinataires des ouvrages chirurgicaux pour mettre en évidence la redéfinition disciplinaire qui s'opère dans la seconde moitié du XVIIIe siècle. Entre 1760 et 1799, la censure puis le simple contrôle des institutions s'accentuent, grâce à la politique de subvention de l'État aux ouvrages scientifiques à partir de 1795. Dans le même temps, le " public », destinataire explicite des ouvrages de chirurgie se transforme : client et juge à l'origine, il est progressivement disqualifié par les chirurgiens, qui en viennent à s'adresser à leurs pairs et à une communauté médicale conçue dans sa totalité sociale, des pairs aux étudiants, public privilégié à la fin du siècle.

Vulgarization and Dissemination of Medecine during the Revolution: the Example of Surgery This study concerns the vulgarization and dissemination of medical knowledge and focuses on the example of surgery. Reviewing the institutional and economic structures of dissemination, it tackles the issue of how the readership of surgical textbooks was constructed and thereby highlights the disciplinary reconfiguration taking place in the second half of the 18th century. Between 1760 and 1799, censorship, then straightforward vetting of institutions gathered momentum, thanks to the policy of state subsidies to scientific publications as from 1795. At the same time, the "public» or explicit addressees of surgical works underwent a change : originally clients and arbiters, they were gradually disqualified by the surgeons who 
now addresssed their peers and a medical community conceived of as a social entity, from peers to students, a privileged audience by the end of the century.

INDEX

Mots-clés : médecine, sociétés savantes, chirurgie, histoire du livre, public

\section{AUTEUR}

CHRISTELLE RABIER

Université Paris I 